Universidad Nacional de La Plata

Facultad de Humanidades y Ciencias de la Educación

Departamento de Sociología

\title{
La internacionalización de las Ciencias Sociales: una reflexión
}

\section{The internationalization of Social Sciences: a reflection}

\author{
Renato Ortiz * \\ * Universidade de Campinas (Unicamp), Brasil | rortiz@terra.com.br \\ Traducción del portugués: Anabel Beliera
}

\section{PALABRAS CLAVE}

\section{RESUMEN}

Internacionalización

El artículo se propone ahondar en la reflexión sobre el proceso de internacionalización de las ciencias sociales contemporáneas. Se reflexiona sobre algunos ejes específicos referidos a cuestiones propias del desarrollo disciplinar -la historia de las Ciencias Sociales y en particular de la Sociología, y el lugar que han tenido los distintos países en la configuración de las tradiciones del pensamiento social-, así como cuestiones extradisciplinares -como las políticas científicas, el rol del Estado y las fuentes de financiamiento. La crítica a la idea de monopolio de lo universal dio lugar a nuevas realidades que exceden la dicotomía norte-sur, y que resaltan la heterogeneidad de las tradiciones intelectuales nacionales. Actualmente nos encontramos ante la creencia en la racionalidad de la evaluación, la cual nace en el interior del campo académico, y resulta de una mentalidad productivista que encuentra en las instituciones universitarias y de investigación el lugar de su realización plena. Esta creencia es parte del proceso de internacionalización de las ciencias sociales y se ha convertido

Historia de las ciencias sociales en sentido común planetario.

\section{KEYWORDS ABSTRACT}

Internationalization

Social Sciences

Social thought

History of the social sciences
This article aims to analyse the process of internationalization of contemporary social sciences. It reflects on some specific issues related to the disciplinary development - social science history, particularly Sociology, and the way in which different countries have shaped the traditions of social thought- as well as some extra-disciplinary topics - such as scientific policies, the role of the State and the financing sources. The critic to the idea about monopolic of universality led to new realities that go beyond the north-south dichotomy, highlighting the heterogeneity of national intellectual traditions. Currently we are facing the growing belief in the rationality of evaluation, which is born within the academic field, and come from a productivist mindset that finds in the university and research institutions the place of its full realization. This belief is part of the internationalization of social sciences internationalization process and has become a world wide common sense. 
El tema de la internacionalización se volvió una cuestión actual en las Ciencias Sociales contemporáneas. Institutos de investigación, organizaciones internacionales (ONU y Unesco), asociaciones científicas nacionales (Sociedad Brasilera de Sociología) e internacionales (International Sociological Association) la ven como un aspecto decisivo en el proceso de circulación de las ideas. En Brasil, la Capes considera la dimensión internacional un criterio relevante en su metodología de evaluación de los cursos de postgrado. Sin embargo, dejo de lado esta dimensión institucional, ya que mi interés es problematizar la cuestión desde otro punto de vista. Un punto de vista, digamos, crítico, porque tiene como foco principal las transformaciones que inciden directamente sobre el trabajo intelectual. Dado que este es un texto corto, organizaré mi reflexión en puntos específicos. Los mismos están condensados y buscan sintetizar una problemática más amplia; tienen la virtud de explicitar determinados aspectos del debate pero son incompletos y merecerían una elaboración mayor de mi parte.

1. Quien estudia el fenómeno de la globalización está familiarizado con la distinción entre globalización e internacionalización. Difícilmente el proceso de globalización podría ser comprendido como resultado de la inter-acción entre las naciones; es necesario descentrarse de la unidad nacional para comprender su lógica. Surge entonces una duda: ¿deberíamos hablar de internacionalización o de una mundialización de las Ciencias Sociales? A primera vista, el término internacionalización sería más adecuado dado que el campo de los cientistas sociales se materializa a través de las instituciones nacionales que le dan soporte. Eso no significa que su consolidación se haga al abrigo de las influencias externas; los intercambios internacionales existen desde el inicio de su constitución en tanto es en la relación con el Estado-Nación que se desarrolla la educación e investigación. En este sentido, hay una estrecha relación entre la expansión de las Ciencias Sociales y las políticas de Estado. En un texto poco conocido, de los inicios de los años 90, Bourdieu (1991) hace esta misma pregunta: ¿existiría un campo mundial de las Ciencias Sociales? Su respuesta es negativa. Dicho campo no tendría la autonomía necesaria para definirse como tal. Johan Heilbron (2013), escribiendo décadas después, dirá que estaríamos asistiendo a su emergencia ahora, cuestión que busca aprender a través de la circulación de revistas e investigadores. Tengo dudas a este respecto. Un campo mundial presupone las exigencias de una autonomía relativa en relación a las demandas de la política (Estado, partido, movimientos sociales) y del mercado; por otro lado, supone una interacción orgánica entre los actores de este espacio de alcance planetario, lo que a mi entender es inexistente $\mathrm{y}$, tal vez, no deba existir. No deja de ser interesante observar que algunos autores hablen, inclusive, de la existencia de un worldsciencesystem, término derivado de los análisis de Wallerstein sobre el sistema mundial. Mi desconfianza en relación a tal perspectiva resulta de la fragilidad estructural de este supuesto del "sistema mundo", dado que el mismo no está compuesto por elementos sistemáticamente articulados entre sí. No obstante, pienso que el proceso de globalización, como en otras esferas, incide también en la configuración de las Ciencias Sociales. El mismo no define propiamente un campo mundial, en el sentido en que el concepto lo entiende, y sin embargo, se constata de hecho el surgimiento de un espacio transnacional que poco a poco se impone. En este sentido, la internacionalización que antes existía se manifiesta ahora en una situación de globalización, o sea, las transformaciones actuales no se resumen en los intereses y reglas existentes en el interior de sus fronteras nacionales.

2. En la constitución de este espacio transnacional, la historia de las disciplinas es importante, 
particularmente en lo que se refiere a la Sociología. Una disciplina implica la organización de la investigación y de la enseñanza según reglas y normas específicas; sus fundamentos se basan en la delimitación de un determinado objeto y de una metodología propia. Es de esta forma que gradualmente la Sociología se destaca de otros saberes en el siglo XIX, imponiéndose poco a poco como un área especializada de la comprensión de lo social. Hay, en consecuencia, una historia de la disciplina. La Sociología surge en algunos países europeos (Francia, Alemania, Inglaterra, Italia) y en los Estados Unidos, para enseguida difundirse en diferentes lugares. En este proceso de expansión internacional hay una clara división del trabajo intelectual. Recuerdo la distinción que Tiryakin (2001) hacía entre “grande” y “pequeña” tradición del pensamiento sociológico. La primera constituiría el eje de la disciplina, alrededor del cual se estructurarían los problemas y argumentos centrales del conocimiento y de la investigación. Cabría a la "pequeña tradición” un papel menor, que resumiría a la investigación orientada a las realidades coyunturales de cada país. El monopolio de lo universal se concentraría en las manos de los herederos de los padres fundadores, a él contrastaría una diversidad de estudios locales, importantes, pero circunscritos a sus localidades. Las verdaderas reflexiones teóricas serían así un atributo de “occidente”, no tanto por una cuestión de discriminación sino simplemente porque la esencia de la modernidad se encontraría en este mismo Occidente (leer por ejemplo la introducción de Weber a "La ética protestante y el espíritu del capitalismo”). Fuera de él, tendríamos una modernidad incompleta, truncada, inacabada. En rigor, un estudio productivo y original de la modernidad periférica sería siempre insatisfactorio; al final, le faltaría la densidad denegada por la historia. El eurocentrismo legitimaba, de esta forma, una convivencia cortés de la división internacional del trabajo intelectual en la que la universalidad del método sería la prerrogativa de algunos, pero su aplicación se encontraría disponible para todos. ${ }^{1}$ Por otro lado, la emergencia de las Ciencias Sociales, particularmente en América Latina, reforzará esa dimensión local. El "pensamiento latinoamericano”, como decía Leopoldo Zea, para liberarse de las amarras extranjeras debe solidificar los lazos de la identidad nacional, volcarse al entendimiento de su propia realidad, aquello que escaparía a las categorías forjadas fuera de su ámbito. Para desarrollarse, las Ciencias Sociales latinoamericanas valorizan lo que les es propio, el rasgo de distinción en relación a los otros. Esto significa que están marcadas por una dualidad: por un lado, está la consolidación efectiva de la disciplina, la creación de universidades e institutos de investigación, la valorización de una tradición de pensamiento; pero por el otro lado, la oposición universal vs local se reitera, pues el dominio del pensamiento se resume a las fronteras de cada país: sociología brasileña, argentina, mexicana, peruana, etc. Lo nacional posibilita la afirmación identitaria pero también distancia del alcance teórico anhelado.

3. El monopolio de lo universal se rompe en la situación de globalización, pues deja de ser un registro de verdad convincente. Hay varios motivos para eso. Primero, una crítica generalizada de la visión eurocéntrica del mundo. Eso se da en el nivel de las ideas, por ejemplo, los escritos de Jack Goody, pero también con el surgimiento de nuevos movimientos teóricos y el reordenamiento disciplinar ocurrido al final del siglo XX: el debate sobre la posmodernidad, la cuestión de la interdisciplinariedad (estudios de género, 
medio ambiente), Estudios Culturales, teorías postcoloniales, etc. En el caso de la Sociología, puede decirse que la versión predominante de la modernidad se torna parcial, se percibe que su validez se limita a un momento de la historia de la disciplina. O como dice Chakrabarty (2000), Europa es apenas una “provincia” del mundo, su geografía posibilita pero también restringe el alcance del pensamiento sociológico. En este sentido, la modernidad deja de ser vista, en su "esencia”, como una virtud intrínseca al mundo occidental; al expandirse, ella se realiza de manera distinta en función de la historia de los lugares. La modernidad no "es", digo, en su Ser, europea, en este caso ella es sólo temporalmente anterior a las otras, lo que no significa que les sea superior. Yo había dicho que la crítica generalizada al eurocentrismo debilitaba la creencia en el monopolio de lo universal. Subrayo generalizada. Con eso quiero decir, la crítica no es nueva, innumerables autores la hicieron antes (Franz Fanon) y, sin embargo, no había adquirido derecho de ciudadanía en el medio científico. La convivencia cortés de la división internacional del trabajo intelectual la contenía como una exageración teórica indebida. Creo que la generalización de la crítica se hace en consonancia con la materialización de una base empírica representada por la maduración de las Ciencias Sociales en la "periferia", maduración que se objetiva en el surgimiento de un campo de interlocutores coherente e institucionalizado. Las políticas de Estado, la asignación de recursos para investigación, la creación de cursos de postgrado, sitúan el saber sociológico en un nivel distinto del que se encontraba en el siglo XX. En este contexto, utilizando una imagen de Walter Mignolo (2009), la “desobediencia epistemológica” se vuelve posible y plausible.

4. ¿Cómo entender las fuerzas que actúan en este espacio transnacional? ¿En qué medida ellas inciden sobre el trabajo intelectual? Una respuesta posible es considerar la asimetría de las relaciones académicas a partir de la oposición entre centro y periferia. Varios autores preconizan una especie de reactualización de los conceptos elaborados por la teoría de la dependencia en los años 60. Existiría así un desequilibrio entre los países acentuando la dependencia académica de la periferia en relación a la metrópoli (Alatas, 2003; Keim, 2011). Al leer con más cuidado esta literatura, vemos que el concepto de imperialismo, implícito en su raciocinio, adquiere una nueva configuración. El mismo no se limita a una nación (por ejemplo, los Estados Unidos), su alcance es más amplio, se habla del predominio de un espacio geográfico polisémico: el norte (muchas veces sinónimo de occidente). Las Ciencias Sociales producidas en este "lugar" tendrían un papel dominante en la estructura jerárquica del pensamiento sociológico. Un texto interesante a este respecto es el Social Science Report 2010, que demuestra de manera empírica esta desigualdad al medir algunos aspectos: dónde son producidas las revistas de Ciencias Sociales, cuál es el grado de coautoría internacional de los artículos escritos en colaboración con autores de diversos países, las citas. La conclusión es clara: hay una internacionalización desigual, los países del norte concentran los mejores resultados en detrimento de los otros. A la categoría norte se opone el sur. El mismo correspondería a los que encuentran marginalizados del centro. El uso del término sur tiene acepciones variadas según los autores, pero la idea principal es la existencia de un espacio aparte dentro del cual se constituiría, por lo menos en potencia, un saber distinto y contrapuesto a su antípoda. $\stackrel{2}{2}$ Ahí se encontrarían las raíces matriciales de un pensamiento 
alternativo. Es en ese sentido que una autora como Raewyn Connell (2007) se dedica a comprender un conjunto de teorías endógenas elaboradas en distintas regiones del mundo. El sur y el norte serían entrelazados por un conjunto de relaciones de poder, articulando intelectuales e instituciones en la metrópoli y en la periferia. ${ }^{3}$ Los análisis sobre la dependencia académica tienen un mérito, pues vuelven explícitas las relaciones de poder que atraviesan el espacio transnacional de las Ciencias Sociales. Eso no es poco. Alcanzan también un punto neurálgico del aparente consenso anterior. Al cuestionar la división del trabajo intelectual se retira de un grupo de privilegiados el monopolio de la reflexión teórica. La distinción entre "gran” y "pequeña” tradición les aseguraba una reserva natural del mercado, se trataba incuestionablemente de los herederos reconocidos de "los clásicos”; al reescribirse la historia de las Ciencias Sociales (es eso lo que se encuentra en disputa) se releva el grado de arbitrariedad de esta narrativa frágil e idealizada. Sin embargo, desde el punto de vista conceptual la solución encontrada me parece poco convincente. Norte y sur son categorías dicotómicas, ¿no sería eso un reduccionismo? No creo que la diversidad de las Ciencias Sociales a escala planetaria pueda ser aprehendida de esta forma, pues las relaciones entre institutos de investigación, universidades, investigadores, son mucho más complejas de lo que se supone. Difícilmente se inscriban en una ecuación simple como centro vs periferia. Norte y sur son todavía denominaciones que sobrentienden la homogeneidad de cada uno de esos polos. ¿Pero la misma existe realmente? En realidad, el norte está compuesto por una heterogeneidad de distintas realidades. El desarrollo de las Ciencias Sociales en Portugal y España es conflictivo y discontinuo porque, entre otras razones, la historia política de estos países es conflictiva. La dictadura de Salazar y Franco siempre fueron hostiles al florecimiento del pensamiento crítico. Algo semejante ocurre en relación al Este europeo. La presencia de conflictos étnicos, el nazismo, la fase soviética, inciden directamente en el plano de las ideas. No se trata simplemente de una cuestión de asignación de recursos, los ejemplos que cité se refieren a una Europa denominada “periférica”. Basta con que consideremos los países nórdicos. Del punto de vista de la capacidad material, ellos de hecho pertenecen a los países ricos, sin embargo ¿tal homología sería válida para las Ciencias Sociales producidas en su interior? ¿Sería Finlandia capaz de establecer un tipo de dominación teórica e intelectual a escala ampliada, como Francia y Alemania ejercen de alguna manera? Puede decirse lo mismo en relación al sur. El mismo está marcado por una heterogeneidad que se deriva de la historia de las Ciencias Sociales en cada país. La Sociología brasilera tiene un destino distinto de la sociología japonesa debido a su recorrido específico. Inclusive en el periodo de su formación e institucionalización (en los años 50), las influencias externas son distintas. En Japón, después de la Segunda Guerra Mundial, prevalece el modelo norteamericano que privilegia las investigaciones cuantitativas; y en Brasil dicha influencia tiene otro matiz, la presencia americana se asocia a la Escuela de Chicago, siendo combinada a la tradición francesa y alemana. En la África Subsahariana el financiamiento de la investigación sociológica por el Estado es una excepción. $\stackrel{4}{\text { Hay }}$ una fuerte dependencia en relación a los recursos del exterior: ONG, donaciones de los gobiernos europeos (Gran Bretaña, Francia, Holanda), fundaciones norteamericanas (Ford, Rockfeller, Mellon, Kellog, etc.). En Brasil, México y Argentina, los recursos son de origen estatal. No estamos hablando de algo menor, las fuentes de 
financiamiento influencian el tipo de investigación realizada. La dicotomía “norte/sur” en el fondo encubre las condiciones reales de existencia del trabajo intelectual, traza una línea divisora que borra la diversidad de sus acentos.

5. Contrariamente a lo que la narrativa predominante en los cursos de Ciencias Sociales enseña, su historia es distinta de la versión canónica. Recientemente una serie de estudios han enfatizado la importancia de la tradición intelectual nacional en su constitución. Donald Levine (1997) identifica diferentes tipos de tradiciones: británica, francesa, alemana, italiana y americana (el marxismo, con su vocación internacionalista, es la única excepción). Cada una de ellas constituye un punto de partida para construirse una pluralidad de problemáticas teóricas que vendrán posteriormente a determinar el legado clásico de la Sociología. Estamos lejos de una visión que consideraba la emergencia de la disciplina como un todo coherente de alcance y validez universal. O, como pensaba Nisbet (1967), que ella se organizaría en torno a un núcleo central de ideas (comunidad, sagrado, autoridad, status, alienación) antes de difundirse a escala internacional. Esta relectura de la historia subraya además otro aspecto: el papel del Estado. $\underline{5}$ Las cuestiones incorporadas en el seno de la disciplina se hacen eco de los temas políticos enfrentados en cada país: por ejemplo, el proceso de unificación en Italia o la ideología republicana en Francia. No nos olvidemos que Durkheim, al volver de su viaje a Alemania, define la Sociología como una "ciencia esencialmente francesa”. La relación entre Ciencias Sociales y nación, tan viva y envolvente en el siglo XIX, fue de esta forma sublimada. Todo pasa como si lo nacional, sinónimo de localismo, fuera una virtud negativa de los países “al margen”. En el contexto europeo y norteamericano, su sublimación se convierte en un imperativo categórico para garantizar la universalidad de la "gran tradición”. La revisión de los orígenes de las Ciencias Sociales nos permite preguntar en qué medida esas tradiciones (en plural) inciden en el espacio trasnacional y en la composición de los campos nacionales de la disciplina. ¿Cuáles son las implicancias de eso?

Al analizar la moda, Bourdieu considera que una de las formas de reproducción y acumulación del capital simbólico se hace a través de la antigüedad de las “marcas”. La distribución desigual del prestigio debe tener en consideración la duración, o sea, la historia del campo de la moda. En este sentido, las "marcas tradicionales” poseen una ventaja, acumulan a lo largo del tiempo un valor superior a las marcas recientes (Dior versus Paco Rabane); la competencia entre ellas será de esta forma asimétrica. Esta no es, evidentemente, la única forma de atesoramiento del capital simbólico, sin embargo, se trata de una dimensión relevante, manifestándose inclusive en otras esferas (en la educación: prestigio de las instituciones tradicionales). Pienso que es posible decir algo semejante en relación a la herencia sociológica. En su origen, ella se construye por tradiciones nacionales, cada una de ellas encierra un capital cultural originario (la disciplina no existía antes) que determina su posición delante de las otras. Durkheim, Weber, Colley, no son simplemente autores con los que eventualmente dialogamos, sino que denotan algo más, forman el significante de un significado más abarcativo, las tradiciones intelectuales que icónicamente representan. La consagración de sus obras e ideas no se limita a sus personalidades, sino que los trasciende, legitimando una herencia geográficamente distinta. Durkheim nos remite a la Sociología 
francesa, los otros respectivamente a la alemana y norteamericana. Los héroes fundadores de la disciplina no se agotan en sus idiosincrasias (son, claro está, importantes) sino que condensan, “en el origen de los tiempos”, un capital simbólico obtenido de sus tradiciones específicas. Este es el valor transmitido a lo largo de las generaciones, asegurando una jerarquía sutil y convincente en el ámbito de las Ciencias Sociales. Mientras tanto, como dice Bourdieu, para perpetuar el capital cultural debe reproducirse, pues sino se debilita, se desvaloriza. El ejemplo de Italia es interesante. En el pasaje del siglo XIX al XX, la disciplina disfruta de un pasado ilustre (Maquiavelo, Vico) que influye en el trabajo de diversos intelectuales: Mosca, Pareto, Labriola. Sin embargo, como observan algunos autores, al analizarse retrospectivamente su trayectoria, se tiene una "frustración notable" (Marotta \& Gregor, 1961). La Sociología fue marcada por la discontinuidad, una serie de interrupciones (fascismo, dificultades de institucionalización) complicaron su destino. Hubo así una desvalorización de su capital inicial, y el mismo no constituye hoy una inversión altamente rentable en la bolsa de los valores sociológicos. Algo similar, pero menos intenso, ocurre con la tradición británica. Sin duda la misma fue relevante en los países colonizados por el imperio británico (cabe discutir) pero, mirándola a partir de otro referente geográfico, se puede afirmar que en América Latina su papel fue secundario. Perry Anderson (1968), un tiempo atrás, escribió un pequeño texto controvertido a este respecto, argumentando que la Sociología británica era poco expresiva al ser comparada con las otras (de los padres fundadores). Su destino había sido opuesto al de la Antropología, la Historia, la Filosofía, la Crítica Literaria. Mientas esas disciplinas constituyeron un prestigio internacional innegable, la Sociología se habría confinado a las fronteras del provincianismo inglés. O como él afirma de manera lapidaria: "Britain -alone of major Western societies- never produced a classical sociology”. El texto de Anderson originó un intenso debate entre los ingleses (Soffer, 1982), aunque, sin entrar en el fondo de la discusión, interesa subrayar que su “intuición” era en parte verdadera. Los “clásicos” de la Sociología británica no viajaron hacia todos los lugares. Dicho de otra forma, su capital simbólico se volvió más restringido que el de los competidores franceses, alemanes y norteamericanos.

6. En la situación de globalización, hay una redefinición del mercado de bienes lingüísticos. Surge una nueva jerarquización de los idiomas en la cual el inglés adquiere una posición central. Por otro lado, el mismo se desenraiza de su origen e incluso de su expansión en la época colonial (Estados Unidos) para constituirse en el idioma de la modernidad-mundo. El número de hablantes extranjeros que manejan el idioma es actualmente superior al de los hablantes nativos. Es esta condición desterritorializada la que le asegura un poder hasta entonces inexistente, o sea, lo disloca de su origen geográfico inicial para el centro de una galaxia de lenguas. Eso no implica el desplazamiento de las lenguas nacionales (en las cuales las Ciencias Sociales, en su mayoría, se hacen), pues continúan y continuarán floreciendo; sin embargo, pasan a integrar un universo en el cual la relación con el inglés es de subalternidad. El espacio transnacional de las Ciencias Sociales es por lo tanto tensionado por una nueva variable, pues el uso de la lengua no es una actividad neutra (hay una ilusión al respecto cuando se habla de "lengua franca”). $\underline{6}$ El principal problema que aparece se refiere a la cuestión de la autoridad. La visibilidad en el campo científico funciona como un 
capital cultural que diferencia a los investigadores. Publicar en inglés y ser citado a escala transnacional asegura el reconocimiento entre los pares. La magia de la noción de citación reside en esta ilación: ella revelaría la esencia del trabajo intelectual (lo que es falso). Consideremos la base de datos Tomson. Entre 1998 y 2007 se puede establecer una distribución de los artículos publicados en función de las áreas geográficas del mundo. Europa - 38,0\%; América del Norte - 52,2\%; Asia - 8,9\%; América Latina - 1,7\%; Oceanía - 4,7\%; África - 1,6\%; CIS - 1,2\% (Gingras \& Mosbah-Natanson, 2010). Una posible lectura puede hacerse a través de la perspectiva centro/periferia. De hecho, hay una concentración de los artículos en dos áreas geográficas: Europa y América del Norte, lo que significa una distribución desigual de las “capacidades”, o sea, de los recursos, entre los países. Sin embargo, la constatación debe ser complementada por otra: la presencia hegemónica del inglés. Cuando se desdobla la distribución de los artículos publicados en función de los idiomas utilizados, el cuadro es otro: inglés - 94,45\%; francés - 1,25\%; alemán - 0,40\%; español - 0,40\%; portugués - 0,08\%; chino - 0.00\%; holandés - 0,01\%; japonés - 0,06\%; polonés - 0,00\%; italiano - 0.01\%. La configuración lingüística en la que se expresan las Ciencias Sociales significa la consolidación de nuevas relaciones de poder. La misma refuerza la posición de países como Estados Unidos e Inglaterra, y países del sur (¿o sería norte?) que utilizan el inglés como Australia; y descalifica las publicaciones de las Ciencias Sociales “al margen”, así como la producción realizada en francés y alemán. En este contexto, el capital simbólico acumulado por las tradiciones francesas y alemana en parte se desvaloriza, el mercado de los bienes lingüísticos de la modernidad-mundo incide sobre el valor que les era antes atribuido.

7. La historia de las políticas científicas es relativamente reciente; está vinculada a las necesidades de planificación del Estado y de la distribución de recursos. La misma involucra dimensiones distintas, grupos de intereses, racionalización de la maquinaria estatal, relación entre las universidades y empresas, etc. Pero hay un elemento común en toda la política científica: un entendimiento previo del sujeto ciencia. Se supone su existencia en singular y que hay que evaluarlo y poner recursos a su disposición. El tema de la evaluación se torna entonces crucial. Este es un debate que tiene lugar sobre todo en los años 60 y 70 en los Estados Unidos. El libro de Ben-Davis The Scientist's Role in Society, estudio comparativo entre los Estados Unidos y otros países, tiene justamente la intención de fundar una política científica de acción. Digo científica, a fin de cuentas una meta-ciencia, o sea, una reflexión que se sitúa "sobre" la ciencia, naturalmente disfrutaría las virtudes de la cientificidad de su objeto (esta es una ilusión tautológica). O, como expone De Solla Price (1965, p. 233) cuando aún escribe en la década del 60: "necesitamos un cuerpo de conocimiento científico que pueda ser la base de las políticas que los gobiernos y los ciudadanos vengan a exigir”. Es dentro de este contexto que la idea de citación se consolida. La misma materializa el aspecto objetivo en el que se expresa la excelencia científica. Objetivo que, por mesurable, hace posible establecer rankings de revistas, autores, departamentos, institutos de investigación, universidades. La medición de la productividad académica es un elemento nuevo en la estructuración de los campos nacionales y del espacio transnacional de las Ciencias Sociales. La misma determina una “evaluación” de lo que es “producido”. Eso significa que la práctica del trabajo intelectual conoce ahora un conjunto de referencias 
racionales que inciden en la estructura valorativa anterior. El "valor" de un autor como Weber no era determinado en función de las citaciones de su obra, por cierto, ignorada en lengua francesa en el momento en que fue elaborada. Los criterios de evaluación son parte de una dominación racional. Su credibilidad deriva de esta dimensión, de la racionalidad del sistema. Se trata de un movimiento que se desarrolla a escala mundial, pues su validez se vuelve incuestionable frente a los criterios aparentemente objetivos que aprehende. La proliferación de rankings determina otro cuadro de la distribución del trabajo intelectual en los campos nacionales; debido a su dimensión estadística los datos pueden ser incluso comparados entre sí. La performance de cada lugar puede ser contrapuesta y jerarquizada en una escala mundial como si fuera realmente plausible ordenar la diversidad existente a partir de una perspectiva numérica. La creencia en la racionalidad de la evaluación no es una dimensión externa al campo de las Ciencias Sociales. En este sentido, ella difiere de la política, de la religión, de los grandes medios de comunicación, dimensiones externas que contrastan con las exigencias de una "vigilancia epistemológica". Por el contrario, ella nace en el interior del campo académico, resulta de una mentalidad productivista que encuentra en las instituciones universitarias y de investigación el lugar de su realización plena. Su generalización a escala transnacional la transforma en un sentido común planetario, un conocimiento a prueba de cualquier duda o cuestionamiento.

\section{Notas}

1 Retomo algunos argumentos que desarrollé en Ortiz (2015). Traducción al español en Ortiz (2014).

$\underline{2}$ Ver Rosa (2014).

$\underline{3}$ Una crítica interesante del libro fue realizada por Burawoy (2015).

4 Ver Mouton (2010).

$\underline{5}$ Ver Wagner (1989).

6 Ver Ortiz (2008). Traducción al español: Ortiz (2009).

\section{$\underline{\text { Referencias bibliográficas }}$}

Alatas, S. F. (2003). Academic dependency and the global division of labour in the social sciences. Current Sociology, 51(6), 599-613.

Anderson, P. (1968). Components of the national culture. New left review, 50, 3.

Bourdieu, P. (1991). Epilogue: on the possibility of a field of world sociology. En J. S. Coleman, 
Theory for a Changing World (pp. 373-387). Ney York: Westview Press.

Burawoy, M. (18 de marzo de 2015). Travelling theory. Recuperado 6 de noviembre de 2017, a partir de https://www.opendemocracy.net/michael-burawoy/travelling-theory

Chakrabarty, D. (2000). Provincializing Europe: Postcolonial thought and historical difference. Princeton: Princeton University Press.

Connell, R. (2007). Southern theory: The global dynamics of knowledge in social science. Polity Press.

Gingras, Y., \& Mosbah-Natanson, S. (2010). Where are social sciences produced? Social Science Report, 47(43.8), 46-1.

Heilbron, J. (2013). The social sciences as an emerging global field. Current Sociology, 62(5), 685703.

Keim, W. (2011). Counter hegemonic currents and internationalization of sociology: Theoretical reflections and an empirical example. International Sociology, 26(1), 123-145.

Levine, D. N. (1997). Visões da tradição sociológica. Rio de Janeiro: Jorge Zahar Editor.

Marotta, M., \& Gregor, A. J. (1961). Sociology in Italy. The Sociological Quarterly, 2(3), 215-221.

Mignolo, W. D. (2009). Epistemic disobedience, independent thought and decolonial freedom. Theory, Culture \& Society, 26(7-8).

Mouton, J. (2010). The state of social science in sub-Saharan Africa (Social Sciences Report). Paris: UNESCO.

Nisbet, R. A. (1967). The sociological tradition. London: Heinemann.

Ortiz, R. (2008). A diversidade dos sotaques:(o inglês e as ciências sociais). Editora Brasiliense.

Ortiz, R. (2009). La supremacía del inglés en las ciencias sociales. Siglo veintiuno ed.

Ortiz, R. (2014). Universalismo y diversidad. Buenos Aires: Prometeo.

Ortiz, R. (2015). As ciências sociais e a diversidade dos sotaques. En Universalismo e Diversidade. San Pablo: Boitempo.

Price, D. J. de S. (1965). The scientific foundations of science policy. Nature, 206(4981), 233-238.

Rosa, M. C. (2014). Sociologias do Sul. Ensaio bibliográfico sobre limites e perspectivas de um campo emergente. Civitas-Revista de Ciências Sociais, 14(1).

Soffer, R. N. (1982). Why do disciplines fail? The strange case of British sociology. The English Historical Review, 97(385), 767-802.

Tyriakian, E. (2001). Traditions in sociology. En International Encyclopaedia of the Social and Behavioral Sciences. Amsterdam: Elsevier.

Wagner, P. (1989). Social-Science and The State In Continental Western-Europe-The Political Structuration of Disciplinary Discourse. International Social Science Journal, 41(4), 509-528. 